\title{
Percutaneous Closure of Patent Foramen Ovale or Atrial Septal Defect in the Presence of Thrombophilia
}

\author{
Subrata Kar, DO', Nabil Noureddin, MD'1, Jamil Aboulhosn, MD², Yakov Mahmzi, BS', \\ Alexandra Coluzzi, BS', Jonathan M. Tobis, MD ${ }^{1 *}$ \\ ${ }^{1}$ Program of Interventional Cardiology, David Geffen School of Medicine, University of California Los Angeles, Los Angeles, California, USA \\ ${ }^{2}$ Ahmanson/UCLA Adult Congenital Heart Disease Center, David Geffen School of Medicine, University of California Los Angeles, Los \\ Angeles, California, USA
}

\begin{abstract}
Background: Some thrombophilic patients have been excluded from clinical trials of septal defect closure due to the presumed higher risk of thrombus formation. The purpose of this study was to assess whether pre-existing thrombophilia predisposes patients to adverse events after percutaneous patent foramen ovale (PFO) or atrial septal defect (ASD) closure.

Methods: A retrospective observational study was performed including 147 patients with thrombophilia who were referred for percutaneous PFO or ASD closure for cryptogenic stroke or transient ischemic attack $(n=104,70.7 \%)$, migraine $(n=30,20.4 \%)$, peripheral embolism $(n=5,3.4 \%)$, right ventricular enlargement $(n=2,1.4 \%)$, desaturation $(n=1,0.7 \%)$, or a combination of diagnoses ( $n=4,2.7 \%$ ). Post-procedure follow-up included 3-6 month clinical evaluation, chart review, or phone call.

Results: Of the 147 patients ( $46 \pm 13$ years of age, follow-up of $43.5 \pm 34.7$ months) who met the inclusion criteria, 142 had a PFO (96.6\%), and 5 had an ASD (3.4\%); $69(46.9 \%)$ underwent closure. Of the closure patients, 1 (1.4\%) developed thrombus (Amplatzer ASO device). There were no cases of endocarditis or device erosion. One patient had a stroke post-closure (1.4\%). Of the 80 women with stroke, TIA, or peripheral arterial embolism, $52(65.0 \%)$ were taking oral contraceptive pills or hormone replacement therapy at the time of the event.
\end{abstract}

(C) 2017 Journal of Structural Heart Disease Published by Science International Corp. ISSN 2326-4004

Accessible online at: http://structuralheartdisease.org/
Conclusions: Percutaneous closure can be safely performed without a significant risk of thrombus formation despite the presence of thrombophilic factors. The most common reversible thrombophilic factor was exogenous estrogen compounds (43\%), whereas $57 \%$ of patients had irreversible thrombophilic disorders.

Copyright @ 2017 Science International Corp.

Key Words

Atrial septal defect - Patent foramen ovale • Thrombophilia - Stroke • Percutaneous closure

\section{Introduction}

Intra-cardiac defects such as patent foramen ovale (PFO) or atrial septal defect (ASD) are often closed to prevent cryptogenic stroke, transient ischemic attack (TIA), migraine, or right ventricular enlargement. The risk of thrombotic formation on the device may be increased in patients with thrombophilia or prothrombotic states [1-3]. The safety and recurrence rate of thrombotic events after PFO or ASD closure in patients with thrombophilia or prothrombotic states are unknown because such patients are excluded from randomized clinical trials. This retrospective observational study of percutaneous PFO or ASD closure in

*Corresponding author:

Jonathan M. Tobis, MD

Program of Interventional Cardiology, David Geffen School of Medicine 757 Westwood Plaza,

Los Angeles, California 90095, USA

Tel.: +1 310794 4797; Fax: +1 310267 0384; E-Mail: jtobis@mednet.ucla.edu 
patients with thrombophilia was performed to determine whether atrial septal device implantation could be performed safely without a high risk of device thrombosis or recurrent thrombotic events.

\section{Materials and Methods}

A retrospective observational study was conducted in a tertiary care center that performs a moderate volume of percutaneous septal closure procedures. A chart review and analysis of the database was performed to determine if any patients who underwent PFO or ASD closure had thrombophilic disorders or prothrombotic states such as gravid state, treatment at the time of presentation with hormone replacement therapy (HRT) or oral contraceptive pills (OCP), or cancer. Hypercoagulability and genetic laboratory testing was performed at our institution or the outside institution that referred the patient for closure. The presence of anticardiolipin antibody, beta-2 glycoprotein antibody, Dilute Russell viper venom time, prothrombin gene 20210A mutation (deoxy-ribonucleic acid test), Factor $V$ Leiden, protein C activity, protein $S$ free antigen assay, antithrombin activity assay, and Factor VIII activity was assessed. The presence of an ASD or PFO was documented by transesophageal echocardiography, transthoracic echocardiography, or transcranial Doppler evaluation and confirmed by crossing the atrial septum at the time of cardiac catheterization.

Risk factors for atherosclerosis, vascular disease, or thrombosis were identified such as hypertension, hyperlipidemia, diabetes, smoking, history of deep venous thrombosis or pulmonary embolism, history of atrial fibrillation or atrial flutter, or history of stroke or TIA (Table 1). Thrombophilic abnormalities consisted of protein $\mathrm{C}$ or $\mathrm{S}$ deficiency, hyperhomocysteinemia, elevated Factor VIII activity, antiphospholipid antibody, vasculitis, gravid state, OCP, HRT, prothrombin gene mutation, beta-2 glycoprotein antibody, Factor V Leiden mutation, antithrombin III deficiency, beta thalassemia minor, or cancer (Table 2).

Pre-device closure treatment consisted of warfarin only, aspirin only, aspirin and plavix, or a combination of warfarin, aspirin, and plavix. Post-closure treatment consisted of aspirin and plavix ( $n=46,66.7 \%$ ), a combination of aspirin, plavix, and warfarin $(n=1$,
Table 1. Patient characteristics (total $=147$ patients)

\begin{tabular}{|c|c|}
\hline Patient Characteristics & n (\%) \\
\hline 1. Age (46 \pm 13 years old) & 147 \\
\hline 2. Females & $117(79.6 \%)$ \\
\hline 3. Diabetes & 0 \\
\hline 4. Hyperlipidemia & $2(1.4 \%)$ \\
\hline 5. Hypertension & $5(3.4 \%)$ \\
\hline 6. Smoker (past/current) & $14(9.5 \%)$ \\
\hline \multirow{2}{*}{$\begin{array}{l}\text { 7. History of deep venous } \\
\text { thrombosis or pulmonary } \\
\text { embolism }\end{array}$} & $14(9.5 \%)$ \\
\hline & $\begin{array}{c}\text { [10 irreversible (71.4\%); } \\
4 \text { reversible group (28.9\%)] }\end{array}$ \\
\hline $\begin{array}{l}\text { 8. history of atrial fibrillation } \\
\text { or atrial flutter }\end{array}$ & $4(2.7 \%)$ \\
\hline \multirow{2}{*}{$\begin{array}{l}\text { 9. history of stroke or } \\
\text { transient ischemic attack }\end{array}$} & $104(70.7 \%)$ \\
\hline & $\begin{array}{c}\text { [55 (52.9\%) reversible; } \\
49 \text { irreversible group (47.1\%) }\end{array}$ \\
\hline
\end{tabular}

$1.4 \%)$, warfarin followed by aspirin and plavix ( $\mathrm{n}=1$, $1.4 \%)$, aspirin and warfarin ( $n=6,8.7 \%$ ), aspirin only in plavix-allergic patients $(n=3,4.3 \%)$, plavix only ( $n$ $=1,1.4 \%)$, or warfarin only $(n=10,14.5 \%)$. One patient's medical regimen post-closure was unknown. The duration of medical treatment was usually 6 months; occasionally, lifelong treatment at the discretion of the consulting hematologist was implemented for thrombophilia.

Patients were informed of the investigational and non-FDA-approved indication for PFO closure and desired closure for prevention of future strokes, complex migraine with transient visual neurologic deficit, or migraine without aura. Informed and written consent was obtained from all patients.

Based upon operator preference, all patients received peri-procedural or post-procedural antibiotics (cefazolin or vancomycin) up to $24 \mathrm{~h}$ after closure. Patient outcomes were assessed via 3-6 month clinical follow-up, chart review, or telephone call (mean, 43.5 \pm 34.7 months). Mortality status was verified by chart review and the Social Security Death Index.

\section{Results}

From the total population of 933 patients with PFO or ASD, 861 had a PFO (92.3\%), and 72 had an 
Table 2. List of hypercoagulable conditions (total = 147 patients)

\begin{tabular}{|c|c|}
\hline $\begin{array}{l}\text { Hypercoagulable Conditions } \\
\text { (18 categories) }\end{array}$ & n (\%) \\
\hline$\S O C P$ & $46(31.3 \%)$ \\
\hline$\neq \mathrm{HRT}$ & $26(16.3 \%)$ \\
\hline Pregnant & $6(4.1 \%)$ \\
\hline$+C A$ & $4(2.7 \%)$ \\
\hline Autoimmune Disease & $27(18.4 \%)$ \\
\hline$\S O C P$ and $\neq H R T$ & $70(47.6 \%)$ \\
\hline $\begin{array}{l}\text { Medication induced (‡HRT, §OCP, or †CA } \\
\text { medications) }\end{array}$ & $72(49.0 \%)$ \\
\hline Beta-thalassemia minor & $1(0.7 \%)$ \\
\hline Elevated lipoprotein A & $1(0.7 \%)$ \\
\hline Protein C or S deficiency & $12(8.2 \%)$ \\
\hline Factor V Leiden mutation & $12(8.2 \%)$ \\
\hline Prothrombin gene mutation & $16(10.9 \%)$ \\
\hline Elevated Factor VIII level & $1(0.7 \%)$ \\
\hline *APS or anti-cardiolipin antibody & $25(17.0 \%)$ \\
\hline Anti-thrombin 3 deficiency & $3(2.0 \%)$ \\
\hline Beta-2-glycoprotein-1 antibody & $9(6.1 \%)$ \\
\hline Presumed hypercoagulable state & $1(0.7 \%)$ \\
\hline Hyperhomocysteinemia & $3(2.0 \%)$ \\
\hline Thrombocytosis & $2(1.4 \%)$ \\
\hline Combined thrombophilia & $44(30.0 \%)$ \\
\hline
\end{tabular}

${ }^{*}$ APS $=$ anti-phospholipid syndrome; $+C A=$ cancer $;$ ҒHRT $=$ hormone replacement therapy; $§ O C P=$ oral contraceptives

ASD (7.7\%). Three of these patients were referred for surgery and excluded. There were 147 subjects (16\%) who met the thrombophilia inclusion criteria and were referred for percutaneous PFO or ASD closure. Table 1 shows the patient characteristics. Patients were divided into two groups: one with reversible causes of thrombophilia such as OCP/HRT use ( $\mathrm{n}=57,38.8 \%)$ or gravid state $(\mathrm{n}=$ $4,2.7 \%)$ and the other with irreversible causes of thrombophilia as listed in Table 2 ( $n=86,58.5 \%)$. In the reversible group, the inciting cause of thrombophilia such as OCP or HRT use was terminated after the clinical event or hypercoagulable state resolved after fetus delivery. Indications for PFO or ASD closure included a history of previous stroke or TIA ( $n=104,70.7 \%)$, migraine $(n=30$,
Table 3. Patient groups, closure indications, and closure devices used (total of 147 patients)

\begin{tabular}{lc}
\hline Patients Groups & $\mathbf{n}(\mathbf{\%})$ \\
\hline Reversible thrombophilia & $61(41.5 \%)$ \\
Irreversible thrombophilia & $86(58.5 \%)$ \\
Percutaneous Septal Closure & $69(46.9 \%)$ \\
Patients declined closure & $43(29.3 \%)$ \\
Not closure candidate or no *PFO found & $34(23.1 \%)$ \\
during cardiac catheterization or Transesoph- & \\
ageal Echo & \\
Unsuccessful closure & $1(0.7 \%)$ \\
Closure Indications & $\mathbf{n}(\%)$ \\
Stroke/transient ischemic attack & $104(70.7 \%)$ \\
Migraine with or without aura & $30(20.4 \%)$ \\
Right ventricular enlargement & $2(1.4 \%)$ \\
Deep venous thrombosis, pulmonary & $5(3.4 \%)$ \\
embolism, or hypercoagulable state & \\
Peripheral arterial embolism (Toe or popliteal & $2(1.4 \%)$ \\
artery embolus) & \\
Desaturation & $1(0.7 \%)$ \\
Combination of indications & $4(2.7 \%)$ \\
Closure Devices Used (total of 69 closures) & $\mathrm{n}(\%)$ \\
Amplatzer & $34(49.3 \%)$ \\
Cribiform & $2.9 \%)$ \\
\hline & \\
Helex & \\
\hline
\end{tabular}

*PFO = patent foramen ovale

$20.4 \%)$, peripheral embolism ( $n=5,3.4 \%)$, right ventricular enlargement ( $n=2,1.4 \%)$, desaturation ( $n=1,0.7 \%)$, or a combination of diagnoses $(n=4,2.7 \%)$. The study included patients from July 2001 to January 2015.

Of the 147 thrombophilic patients $(46 \pm 13$ years of age), 142 had a PFO (96.6\%), 5 had an ASD (3.4\%), and $69(46.9 \%)$ underwent closure. Forty-three patients $(29.3 \%)$ declined percutaneous closure. Thirty-three patients (22.4\%) were deemed inappropriate candidates for closure or were found to have no PFO during cardiac catheterization ( $n=8,5.4 \%)$. One patient $(0.7 \%)$ underwent transesophageal echocardiography for clarification of a suspected PFO, which revealed the absence of a PFO. All septal closures were successful except 1 ASD case (1.4\%), which was 
unsuccessful secondary to bilateral femoral venous obstruction. Twenty-five patients in the reversible thrombophilic group underwent closure (36.2\%), whereas 44 patients in the irreversible thrombophilia group underwent closure (63.8\%). Only 1 patient $(1.4 \%)$ in the irreversible thrombophilia group had a recurrent stroke after PFO closure. Table 3 presents the patient groups, closure indications, and closure devices used.

One patient in the PFO group $(0.7 \%)$ with anti-cardiolipin antibody and beta-2 glycoprotein antibody experienced pericarditis post-procedure with a small pericardial effusion from suspected nickel allergy (confirmed via patch testing) 1 month after Amplatzer device deployment. One patient (1.4\%) out of the total 34 patients with Amplatzer implants (49\%) developed a small left atrial thrombus on the device 1-month post-implant. This patient had thrombophilia from beta- 2 glycoprotein antibody and was treated post-closure with aspirin and plavix. None of the 33 patients with a Gore Helex device developed thrombus formation. Of the 69 patients who underwent closure, 50 patients $(72.5 \%)$ received antiplatelet medication post-procedure (aspirin only, aspirin and plavix, or plavix only), $10(14.5 \%)$ received an anticoagulant only (warfarin), and $8(11.6 \%)$ received a combination of antiplatelets and an anticoagulant. One patient's post-closure medical regimen was unknown.

One patient developed transient tongue and right lower extremity paresthesia post-closure, and another patient experienced a transient neurologic deficit 1 month post-closure; both of these episodes were believed to be complex migraines without headache.

Of the 117 women (80.0\%), 70 (59.8\%) were receiving HRT or OCP at the time of their event (HRT: $n=24$, 20.5\%; OCP: $n=46,39.3 \%)$, of which 52 (74\%) developed stroke, TIA, or peripheral arterial embolism. Of the 104 patients who had a stroke, TIA, or peripheral arterial embolus, 79 were females (76\%), of which $52(66 \%)$ were taking OCP $(n=34,43 \%)$ or HRT $(n=$ $18,23 \%)$. Of the 70 women on OCP or HRT, 68 (97\%) stopped pre- or post-closure based on the treating physician's suggestion (only 2 patients wanted to continue OCP or HRT post-closure). None of the 70 patients who had been on hormone therapy prior to PFO closure developed recurrent thrombotic events, endocarditis, or device erosion.
Three deaths (2.0\%) occurred, but none were related to the device or implantation. One patient died secondary to myocardial infarction, and 2 deaths were non-cardiac. Of the 2 non-cardiac deaths, 1 patient did not undergo device closure, and the other died from septic shock.

\section{Discussion}

The purpose of this study was to assess whether patients with thrombophilia have an increased risk for stroke or thrombus formation on closure devices following implantation of this foreign body, which has constant contact with circulating blood, in the atrial septum [1]. Due to this hypothetical concern, thrombophilic patients are usually excluded from clinical trials of percutaneous PFO or ASD closure. Nevertheless, clinical indications for closure of ASD or PFO may arise in subjects with thrombophilic conditions. This study included patients with a variety of thrombophilic disorders and prothrombotic states, which has not been reported previously in the literature.

A prospective case-controlled study by Pezzini et al. [1] showed that thrombophilia in PFO patients may pose a risk for stroke. A study by Chaturvedi [3] reported that patients with a PFO and cryptogenic stroke in the presence of thrombophilia had an increased risk of recurrent stroke. They proposed that PFO closure in such patients prevents recurrences.

A case report of a patient with Factor $V$ Leiden and PFO describes a paradoxical coronary embolism that caused myocardial infarction [4]. Factor V Leiden has been associated with increased risk of arterial and venous thrombosis [5]. A second case report described a patient with Factor V Leiden and PFO with subsequent multiple embolic events [6]. A study by Giardini et al. [7] of PFO closure in 72 patients, of whom 20 had thrombophilia, suggests that such patients have enhanced risk of recurrent stroke after a cryptogenic stroke, which is mitigated by PFO closure.

Autoimmune disease may promote a prothrombotic state that could lead to thrombotic events [5]. The prevalence rates of anti-cardiolipin antibodies in patients with ischemic strokes is reported to be $17-21 \%$ [8]. A retrospective case series of 40 patients showed that anti-phospholipid antibodies and hypercoagulability are common in patients with PFO who 
present with cryptogenic stroke [9]. A case-controlled study also showed that anti-phospholipid antibodies are strongly associated with PFO and atrial septal aneurysms [10]. Therefore, autoimmune diseases with various hypercoagulable factors may increase the risk of stroke. However, the PFO in Cryptogenic Stroke Study and Anti-Phospholipid Antibodies and Stroke Study (PICSS-APASS) post-hoc retrospective analysis did not show an increased risk of stroke in patients with anti-phospholipid antibodies and PFO [11].

Our retrospective observational study shows that PFO or ASD closure can be safely performed in patients with thrombotic disorders or prothrombotic states using the Amplatzer or Gore Helex device. Only 1 thrombotic event occurred on an Amplatzer device (1.4\%) for PFO closure, but this was in the presence of new onset atrial fibrillation. This patient also had hyperhomocysteinemia and experienced recurrent stroke 8 years after closure.

There were no cases of thrombosis observed on Gore Helex devices, which suggests that the e-PTFE device may not be thrombogenic even in thrombophilic patients. In this higher-risk patient population, there was no procedural mortality, systemic infection, endocarditis on the implanted device, or erosion with a mean follow-up of $43.5 \pm 34.7$ months. Thus, septal closure in this thrombophilic patient population may be safely performed with a low risk of recurrent thrombotic events using either Amplatzer or Gore Helex devices.

Of the thrombophilic conditions, most were due to estrogen-containing medications, which are known to increase thrombosis [12]. A higher than expected number ( $n=79$ out of $104 ; 76 \%$ ) of patients who had a stroke, TIA, or peripheral arterial embolus were females, of whom 51 (65\%) were receiving OCP or HRT. These observations suggest that estrogen compounds predispose to paradoxical embolism in the presence of a septal defect. These findings are consistent with those of our previous study showing a higher rate of PFO in post-menopausal women with a stroke who were using HRT [13]. Stopping OCP or HRT may have led to the low rate of recurrent stroke following septal closure, but this was not a randomized trial of medical therapy (without OCP) versus device closure. It is not known what the recurrent stroke rate would be in women who stop taking hormone therapy but do not get their PFO closed. This raises the question whether enrolling women who were on OCPs at the time of the event but subsequently stopped taking OCPs might bias the population of PFO closure for cryptogenic stroke trials to a lower risk group and thus produce lower subsequent events.

The Randomized Evaluation of Recurrent Stroke Comparing PFO Closure to Established Current Standard of Care Treatment (RESPECT) trial [14] showed a 10-year event rate point estimate for annualized recurrent stroke in the intention-to-treat cohort of 0.57 per 100 patient-years (closure group) versus 1.05 per 100 patient-years in the medical group. Patients with mild forms of thrombophilia were included in the RESPECT trial, but the investigators did not capture the frequency of OCP/HRT use at the time of the initial stroke. Thus, this large randomized trial did not show whether the lower than expected frequency of recurrent stroke was partly due to stopping hormone therapy at entry into the trial. The RESPECT trial also demonstrates that a longer-term follow-up is necessary, as the risk of recurrent stroke in the medically treated group continued to be constant at about 1\% per year for the 10 years of observation. It is hypothesized that this yearly risk of recurrent stroke might continue in the medically treated group for the rest of the patients' lives.

This was a retrospective observational study of a complex patient population in a tertiary care center, which may limit its generalizability. The procedures were performed in a single center by experienced operators who routinely perform PFO or ASD closures. Nevertheless, this study provides useful data on the management of such complex patients, as most are excluded from randomized clinical trials. Thus, scant information is available about device implantation in patients with thrombophilia.

In conclusion, in this group of patients with thrombophilic conditions, PFO or ASD closure may be safely performed using an Amplatzer or Gore Helex device without a high risk of developing thrombus on the device. Moreover, $66 \%$ of women with thrombophilia who presented with cryptogenic stroke, TIA, or peripheral arterial embolism were receiving OCP or HRT, which is a form of thrombophilia that can be reversed by medication cessation. The high frequency of this transient and reversible form of thrombophilia may 


\section{help explain the low recurrence rate of stroke after an Conflict of Interest}

initial cryptogenic stroke.

The authors have no conflict of interest relevant to this publication.

\section{Comment on this Article or Ask a Question}

\section{References}

1. Pezzini A, Del Zotto $E$, Magoni $M$, Costa $A$, Archetti S, Grassi M, et al. Inherited thrombophilic disorders in young adults with ischemic stroke and patent foramen ovale. Stroke. 2003;34:28-33. PMID: 12511746

2. Florez JC, Ay H, Van Cott EM, Buonanno FS. Patent foramen ovale and hypercoagulability as combined risk factors for stroke. J Stroke Cerebrovasc Dis. 2003;12:114-118. DOI: 10.1016/S1052-3057(03)00041-7

3. Chaturvedi S. Coagulation abnormalities in adults with cryptogenic stroke and patent foramen ovale. J Neurol Sci. 1998;160:158-160. PMID: 9849798

4. Croft AP, Khan JN, Chittari MV, Varma C. Paradoxical coronary artery embolism causing acute myocardial infarction in a young woman with factor $\mathrm{V}$ Leiden thrombophillia. J R Coll Physicians Edinb. 2012;42:218220. DOI: 10.4997/JRCPE.2012.306

5. Saver JL. Emerging risk factors for stroke: patent foramen ovale, proximal aortic atherosclerosis, antiphospholipid antibodies, and activated protein C resistance. J Stroke Cerebrovasc Dis. 1997;6:167-172. PMID: 17894989

6. Tang L, Fang ZF, Zhou SH. Paradoxical embolism causing acute embolic events in a patient with hereditary thrombophilia. Herz. 2015;40:314-317. DOI: 10.1007/ s00059-013-3994-9
7. Giardini $A$, Donti $A$, Formigari $R$, Bronzetti G, Prandstraller D, Bonvicini M, et al. Comparison of results of percutaneous closure of patent foramen ovale for paradoxical embolism in patients with versus without thrombophilia. Am J Cardiol. 2004;94:10121016. DOI: 10.1016/j.amjcard.2004.06.056

8. Bushnell CD, Goldstein LB. Diagnostic testing for coagulopathies in patients with ischemic stroke. Stroke. 2000;31:30673078. PMID: 11108774

9. Dodge SM, Hassell K, Anderson CA, Keller J, Groves B, Carroll JD. Antiphospholipid antibodies are common in patients referred for percutaneous patent foramen ovale closure. Catheter Cardiovasc Interv. 2004;61:123-127. DOI: 10.1002/ccd.10754

10. Tomomasa R, Yamashiro K, Tanaka R, Hattori N. Cerebral infarction in an HIV-infected patient with combined protein $\mathrm{S}$ and $\mathrm{C}$ deficiency and a patent foramen ovale. J Stroke Cerebrovasc Dis. 2013;22:e650-e652. DOI: 10.1016/j.jstrokecerebrovasdis.2013.04.019

11. Rajamani K, Chaturvedi S, Jin Z, Homma $\mathrm{S}$, Brey RL, Tilley $\mathrm{BC}$, et al. Patent foramen ovale, cardiac valve thickening, and antiphospholipid antibodies as risk factors for subsequent vascular events: the PICSSAPASS study. Stroke. 2009;40:2337-2342. DOI: $10.1161 /$ STROKEAHA.108.539171
12. Rosendaal FR, Helmerhorst FM, Vandenbroucke JP. Female hormones and thrombosis. Arterioscler Thromb Vasc Biol. 2002;22:201-210. PMID: 11834517

13. Greep NC, Liebeskind DS, Gevorgyan R, Truong T, Cua B, Tseng $\mathrm{CH}$, et al. Association of ischemic stroke, hormone therapy, and right to left shunt in postmenopausal women. Catheter Cardiovasc Interv. 2014;84:479-485. DOI: 10.1002/ccd.25431

14. Carroll JD, Saver JL, Thaler DE, Smalling RW, Berry S, MacDonald LA, et al. Closure of patent foramen ovale versus medical therapy after cryptogenic stroke. N Engl J Med. 2013;368:1092-1100. DOI: 10.1056/ NEJMoa1301440

Cite this article as: Kar S, Noureddin N, Aboulhosn J, Mahmzi Y, Coluzzi A, Tobis JM. Percutaneous Closure of Patent Foramen Ovale or Atrial Septal Defect in the Presence of Thrombophilia. Structural Heart Disease. 2017;3(5):135-140. DOI: https://doi. org/10.12945/j.jshd.2017.018.17 